\title{
МЕТОД ФУНКЦИЙ ГРИНА ДЛЯ РАСЧЕТА ПРИМЕСНЫХ СОСТОЯНИИ В НЕОРТОГОНАЛЬНОМ БАЗИСЕ
} S. REIFMAN. GREENI FUNKTSIOONIDE MEETOD LISANDISEISUNDITE MAARAMISEKS MITTE-
ORTOGONAALSES BAASIS

S. REIFMAN. THE GREEN FUNCTION METHOD FOR IMPURITY STATES WITH NONORTHOGONAL BASIS SET

Общим методом расчета одноэлектронного спектра кристалла с дефектом в случае, когда возмущение, создаваемое дефектом, локализовано в малой области кристалла, является метод функций Грина. Этот метод, впервые примененный к проблеме электронных состояний Г. Костером и Дж. Слэтером $\left[{ }^{1}\right]$, позволяет найти как расположение уровней дефекта относительно зон кристалла, так и его волновые функции. В дальнейшем' развитию метода функций Грина применительно к электронной задаче было посвящено много работ (см. обзоры $\left.\left[{ }^{2,3}\right]\right)$.

При применении метода обычно исходят из ортогонализованного базиса волновых функций, локализованных на узлах кристаллической решетки. В $\left[{ }^{4,5}\right]$ - это функции Ванье, в $\left[{ }^{6,7}\right]$ - атомные орбитали, ортогонализованные по Лёвдину. С другой стороны, в современных квантово-химических расчетах электронной структуры используется неортогональный базис, что затрудняет их согласование с методом функций Грина. Кроме того, коэффициенты разложения волновых функций по ортогонализованным узельным функциям не имеют прямого физического смысла и плохо поддаются интерпретации. В связи с этим целесообразно модифицировать метод функций Грина с учетом неортогональности базиса. В настоящей работе проделана такая модификация.

В приближенин МО ЛКАО волновая функция кристалла с дефектом может быть записана в виде

$$
\psi(\mathbf{r})=\sum_{l} c_{l} \varphi_{l}(\mathbf{r}) .
$$

Здесь $\varphi_{l}(\mathbf{r})$ - атомные волновые функции. Индексом $l$ мы обозначили для упрощения записи как номер узла решетки, так и номер атомной орбитали на узле.

Условие нормировки полной функции имеет вид

$$
\sum_{l l^{\prime}} c_{l}^{*} S_{l l^{\prime}} c_{l^{*}}=1
$$

где $S_{l l^{\prime}}=\left\langle\varphi_{\imath} \mid \varphi i^{\prime}\right\rangle-$ интегралы перекрывания. 
Одноэлектронное уравнение в нашем приближении можно записать следующим образом:

$$
\sum_{u^{\prime}}\left(F_{l l^{\prime}}-E S_{l l^{\prime}}\right) c_{l^{\prime}}=0
$$

Здесь $F_{l^{\prime}}$ - матричные элементы оператора Фока. Отвлекаясь от вопроса о самосогласовании, предположим, что этот оператор известен и что задача сводится к нахождению одноэлектронных энергий и волновых функций $c_{l}$. Допустим также, что нам известны оператор Фока $\mathbf{F}^{0}$ и матрица интегралов перекрывания $\mathbf{S}^{0}$ для идеального кристалла. Перепишем уравнение (3) в виде

$$
\sum_{l^{\prime}}\left[F_{l l^{\prime}}^{0}+\Delta \mathbf{F}_{l l^{\prime}}-E\left(S_{l l^{\prime}}^{0}+\Delta S_{l l^{\prime}}\right)\right] c_{l^{\prime}}=0 .
$$

Здесь $\Delta \mathrm{F}$ и $\Delta \mathrm{S}$ - возмущения оператора Фока и матрицы перекрывания, вносимые дефектом. Допустим, что элементы матриц возмущения отличны от нуля лишь в малой области $\mathbf{L}$ вблизи дефекта.

Разложим волновую функцию кристалла с дефектом по полному набору собственных функций невозмущенной задачи:

$$
c_{l}=\sum_{\mathbf{k} j} D_{\mathbf{k} j} c_{l}^{0}(\mathbf{k j}),
$$

где $\mathbf{k}-$ волновой вектор, $j$ - номер зоны. Волновые функции идеального кристалла $c_{l}{ }^{0}(\mathbf{k} j)$ удовлетворяют уравнению

$$
\left[\mathrm{F}^{0}-E(\mathbf{k j}) \mathrm{S}^{0}\right] c^{0}(\mathbf{k} j)=0
$$

и обладают свойствами ортонормированности и полноты

$$
\begin{aligned}
& \sum_{l^{\prime}} c_{l}^{0^{*}}(\mathbf{k} j) S_{l^{\prime}}^{0} c_{l^{\prime}}^{0}\left(\mathbf{k}^{\prime} j^{\prime}\right)=\delta_{\mathbf{k} \mathbf{k}^{\prime}} \delta_{j j^{\prime}}, \\
& \sum_{\mathbf{k} j l^{\prime \prime}} c_{l}^{0 *}(\mathbf{k} j) S_{l^{\prime} l^{\prime}}^{0} c_{l^{\prime \prime}}^{0}(\mathbf{k} j)=\delta_{l^{\prime}} .
\end{aligned}
$$

Подставляя выражение (5) в (4), домножая (4) на $c_{l}{ }^{0 *}(\mathbf{k} j)$ и суммируя по $\mathbf{k}$ и $j$, получим с учетом (7):

$$
D_{\mathrm{k} j}=\sum_{u^{\prime}} \frac{c_{l}^{0^{*}}(\mathrm{k} j)\left(\Delta F_{l^{\prime}}-E \Delta S_{l^{\prime}}\right) c_{l^{\prime}}}{E-E^{0}(\mathrm{k} j)} .
$$

Комбинируя (8) и (5), получим следующую систему уравнений:

где

$$
c_{l}=\sum_{l l^{\prime \prime}} G_{l l^{\prime}}\left(\Delta F_{l^{\prime} l^{\prime \prime}}-E \Delta S_{l^{\prime} l^{\prime \prime}}\right) c_{l^{\prime \prime}}
$$

$$
\mathbf{G}_{l^{\prime}}(E)=\sum_{\mathbf{k} j} \frac{c_{l}^{0 *}(\mathrm{kj}) c_{l^{\prime}}^{0}(\mathrm{k} j)}{E-E^{0}(\mathrm{k} j)}
$$

- элементы функции Грина $\mathbf{G}=\left(E \mathbf{S}^{0}-\mathbf{F}^{0}\right)^{-1}$ в узельном представлении. Формула (10) в точности совпадает с формулой для матричных элементов функции Грина в случае ортогонального базиса.

Если в (9) ограничиться узлами $l, l^{\prime}$, принадлежащими дефектной области $\mathbf{L}$, получится замкнутая однородная система уравнений, условие разрешимости которой

$$
\operatorname{det}\left|\delta_{u^{\prime \prime}}-\sum_{l^{\prime}} g_{u^{\prime}}\left(\Delta F_{l^{\prime} l^{\prime \prime}}-E \Delta S_{l^{\prime} l^{\prime \prime}}\right)\right|=0
$$


где $g_{u^{\prime}} \equiv G_{l^{\prime}}$ при $l, l^{\prime} \in \mathbf{L}$, определяет энергии локальных состояний.

Для того чтобы найти волновые функции локальных состояний, перепишем (2) следующим образом:

$$
\sum_{u^{\prime}} c_{l}^{*}(t) S_{l l^{\prime}}^{0} c_{l^{\prime}}(t)+\sum_{l l^{\prime}} c_{l}^{*}(t) \Delta S_{l^{\prime}} c_{l^{\prime}}(t)=1
$$

где $t$ нумерует локальные состояния. Во вторую сумму выражения (12) дают вклад только члены с $l, l^{\prime} \in \mathbf{L}$. Для преобразования первой суммы подставим в нее выражения для $c_{t}$ из формулы (9). Тогда, учитывая, что

$$
\sum_{m m^{\prime}} G_{l m}(E) S_{m m^{\prime}}^{0} G_{m^{\prime} l^{\prime}}(E)=-\frac{\mathrm{d}}{\mathrm{d} E} G_{l l^{\prime}}(E)
$$

получим

$$
\begin{gathered}
\sum_{u^{\prime}} c_{l}^{*}(t) \Delta S_{u^{\prime}} c_{l^{\prime}}(t)-\sum_{u^{\prime}} \sum_{m m^{\prime}}\left(\Delta F_{l^{\prime}}-E \Delta S_{l l^{\prime}}\right) \frac{\mathrm{d} g_{l m}}{\mathrm{~d} E} \times \\
\times\left(\Delta F_{m m^{\prime}}-E \Delta S_{m m^{\prime}}\right) \dot{c}_{l^{\prime}}(t) c_{m^{\prime}}(t)=1 .
\end{gathered}
$$

Наконец, еще раз используя (9) и учитывая, что для любой неособенной матрицы справедливо соотношение

$$
\mathrm{g}^{-1}(E)\left[\frac{\mathrm{d}}{\mathrm{d} E} \mathrm{~g}(E)\right] \mathrm{g}^{-1}(E)=-\frac{\mathrm{d}}{\mathrm{d} E} \mathrm{~g}^{-1}(E),
$$

получим

$$
\sum_{u l^{\prime}} c_{l}^{*}(t)\left\{\left.\frac{\mathrm{d}}{\mathrm{d} E}\left[g^{-1}(E)\right] u_{u^{\prime}}\right|_{E=E_{t}}+\Delta S_{u^{\prime}}\right\} c_{l^{\prime}}(t)=1,
$$

где $E_{t}$ - энергия локального состояния. Выражение (13) и уравнение (9) позволяют определить коэффициенты разложения волновой функции локального состояния сначала в дефектной области, а затем и во всем кристалле.

Автор глубоко признателен Г. С. Завту и Б. В. Шуличенко за обсуждение результатов работы.

\section{ЛИ Т Е Р А Т Р А}

1. Koster, G. F., S1ater, J. C. Wave functions for impurity levels. - Phys. Rev., 1954 , v. 95 , N 5, p. $1167-1176$.

2. Р ой и н Ӓ. Б. Теория глубоких центров в полупроводниках. - ФТП, 1974, т. 8, № 1 , c. $3-29$.

3. B assani, F., I a donisi, G., Preziosi, B. Electronic impurity levels in semiconductors. - Rep. Prog. Phys., 1974, v. 37, N 9, p. 1099-1210.

4. C a 11 a way, J., $\mathrm{Hu}$ ghes, A. J. Localized defects in semiconductors, - Phys. Rev., 1967 , v. 156 , N 3, p. $860-876$.

5. F a u $1 \mathrm{kner}$, R. A. Toward a theory of isoelectronic impurities in semiconductors. Phys. Rev., 1968, v. 175 , N 3, p. 991-1009.

6. C a 1 a is, J.-L., R ibbing, C.-G. Use of orthogonalized atomic orbitals in the Koster-Slater method for impurities, - Phys. Rev., 1971, v. B4, N 2, p. 376382.

7. 3 а в т Г. Локальные одноэлектронные состояния в модели сильной связи. - Изв. АН ЭССР, Физ. Матем., 1975 , т. 24 , № 1, с. 92-106.

Тартуский государственный университет
Поступила в редакцию 19/XII 1977 